University of Nebraska - Lincoln

DigitalCommons@University of Nebraska - Lincoln

September 2000

\title{
The RR Lyrae Star V442 Herculis: An Extreme Case of Light-Curve Modulation
}

\author{
Edward G. Schmidt \\ University of Nebraska-Lincoln, eschmidt1@unl.edu \\ Kevin M. Lee \\ University of Nebraska-Lincoln, klee6@unl.edu
}

Follow this and additional works at: https://digitalcommons.unl.edu/physicsschmidt

Part of the Physics Commons

Schmidt, Edward G. and Lee, Kevin M., "The RR Lyrae Star V442 Herculis: An Extreme Case of Light-Curve Modulation" (2000). Edward Schmidt Publications. 7.

https://digitalcommons.unl.edu/physicsschmidt/7

This Article is brought to you for free and open access by the Research Papers in Physics and Astronomy at DigitalCommons@University of Nebraska - Lincoln. It has been accepted for inclusion in Edward Schmidt Publications by an authorized administrator of DigitalCommons@University of Nebraska - Lincoln. 


\title{
The RR Lyrae Star V442 Herculis: An Extreme Case of Light-Curve Modulation
}

\author{
E. G. SCHMIDT AND K. M. LeE \\ Department of Physics and Astronomy, University of Nebraska, Lincoln, NE 68588-0111; eschmidt1@unl.edu, klee6@unl.edu \\ Received 2000 February 10; accepted 2000 May 5
}

\begin{abstract}
We have obtained photometric observations of the peculiar RR Lyrae star V442 Her during six observing seasons spanning an elapsed time of nearly 9 years. The period has undergone two large, abrupt changes in the past 5 years. Although light-curve modulation is fairly common among RR Lyrae stars, the modulation of the light curve of V442 Her is highly unusual for its large amplitude, long period, extreme alterations in light-curve shape, and large period changes.
\end{abstract}

\section{INTRODUCTION}

We have undertaken a survey of 107 Bailey type ab RR Lyrae stars to determine the frequency and characteristics of multiperiodic behavior among such objects. Multiperiodicity will be identified by observing the stars near the phase of maximum light and identifying those in which the magnitude of maximum does not repeat well from one cycle to another. As of now, observations have been finished for about two-thirds of the survey sample. Rather than await the conclusion of the survey, results for interesting individual stars from the sample will be published as they are completed. This is the first in a series of such papers.

Although V442 Her is classed as a Bailey type ab RR Lyrae star in the General Catalogue of Variable Stars (GCVS; Kholopov 1985), there is a note indicating that the shape of the light curve varies. A light curve obtained by Schmidt (1991; see also Schmidt \& Reiswig 1993) exhibited an amplitude of only a few tenths of a magnitude, much too small for such a star. Furthermore, the shape of the light curve did not resemble either a Bailey type ab or type c RR Lyrae. Subsequently, Lee (1997) included V442 Her in his survey of light-curve modulation among RR Lyrae stars. His observations during 1995 produced a light curve similar in shape and amplitude to that from the earlier observations. In both 1991 and 1995 there was evidence of significant changes in the variation over times of 2 or 3 months. We have monitored this star since that time and now have observations in six seasons spanning nearly 9 years. This paper presents all those observations and demonstrates the extreme behavior of this star compared with other RR Lyrae stars.

\section{OBSERVATIONS}

Photometry in the $V$ and $R$ bands was obtained with the CCD photometry system on the Behlen Observatory $0.76 \mathrm{~m}$ telescope. The methods used in obtaining and analyzing the data were the same as described earlier (Schmidt 1991 and references therein). In the case of the present observations, there were three comparison stars, of which two were used for most of the observations. They are listed in Table 1, where column (1) identifies the star and column (2) gives its number in the Hubble Space Telescope Guide Star Catalogue (GSC) (in the format rrrrnnnn, where rrrr is the small region of the GSC and nnnn is the star number in that region). Columns (3)-(8) contain the mean magnitudes and colors of the stars and their standard errors (in millimagnitudes). Column (9) indicates the number of nights which were used in forming the mean magnitudes and colors. For stars $\mathrm{C} 1$ and $\mathrm{C} 2$, these numbers refer to photometric nights with adequate standard stars for all-sky photometry. Star C3 was not observed on such nights, and its magnitudes and color in Table 1 are based on differential measurements relative to $\mathrm{C} 1$. The uncertainty of the individual light-curve points depends on a variety of factors including sky conditions, exact location on the CCD chip, and which comparison stars were used. We conservatively estimate from the comparison stars that the uncertainties are generally less than $0.02 \mathrm{mag}$ for both $V$ and $R$.

Table 2 summarizes the data we have obtained. Column (1) lists the year of the observations, while column (2) gives the range of modified Heliocentric Julian Date $(\mathrm{MHJD}=\mathrm{HJD}-2,440,000)$ over which the data were obtained. Columns (3) and (4) list the number of nights on which observations were obtained and the number of individual light-curve points obtained. The individual observations of the variable stars have been placed in the IAU Archives of Unpublished Variable Star Observations as file 340E. See Breger (1990) for a description of the archives and how to access data in them.

Within each season we have divided the data into between two and five subsets. The subsets were selected to span an interval short enough to encompass little change in 
TABLE 1

\begin{tabular}{|c|c|c|c|c|c|c|c|c|}
\hline \multicolumn{9}{|c|}{ COMPARISON STARS } \\
\hline $\begin{array}{l}\text { Star } \\
(1)\end{array}$ & $\begin{array}{c}\text { GSC Number } \\
\text { (2) }\end{array}$ & $\begin{array}{c}V \\
\text { (mag) } \\
(3)\end{array}$ & $\begin{array}{l}\text { s.e. } \\
\text { (mag) } \\
(4)\end{array}$ & $\begin{array}{c}R \\
\text { (mag) } \\
(5)\end{array}$ & $\begin{array}{c}\text { s.e. } \\
\text { (mag) } \\
(6)\end{array}$ & $\begin{array}{c}V-R \\
(\mathrm{mag}) \\
(7)\end{array}$ & $\begin{array}{l}\text { s.e. } \\
\text { (mag) } \\
(8)\end{array}$ & $\begin{array}{l}N \\
(9)\end{array}$ \\
\hline $\mathrm{C} 1 \ldots \ldots$ & .31112054 & 14.101 & 8 & 13.595 & 9 & 0.508 & 9 & 9 \\
\hline $\mathrm{C} 2 \ldots \ldots$ & .31111434 & 12.677 & 6 & 12.602 & 9 & 0.076 & 6 & 11 \\
\hline $\mathrm{C} 3 \ldots \ldots$ & .31110957 & 15.108 & 3 & 14.735 & 5 & 0.374 & 4 & $44^{\mathrm{a}}$ \\
\hline
\end{tabular}

a There were no photometric nights on which $\mathrm{C} 3$ was observed. The mean magnitudes and color are based on differential measurements relative to $\mathrm{C} 1$.

the light curve while still containing an adequate number of data with adequate phase coverage to define the light curve. The subsets are listed in Table 3, where column (1) gives the year of the observations and column (2) displays the range of MHJD included in each subset. Column (3) indicates the plot symbol for that subset in Figure 2. The number of

TABLE 2

LOG OF OBSERVATIONS

\begin{tabular}{cccc}
\hline \hline $\begin{array}{c}\text { Year } \\
(1)\end{array}$ & $\begin{array}{c}\text { MHJD Range } \\
(2)\end{array}$ & $\begin{array}{c}\text { Number of } \\
\text { Nights } \\
(3)\end{array}$ & $\begin{array}{c}\text { Number of } \\
\text { Observations } \\
(4)\end{array}$ \\
\hline $1991 \ldots \ldots$. & $8102-8192$ & 11 & 26 \\
$1995 \ldots \ldots$. & $9871-10,002$ & 10 & 362 \\
$1996 \ldots \ldots$. & $10,233-10,357$ & 20 & 294 \\
$1997 \ldots \ldots$. & $10,655-10,796$ & 15 & 171 \\
$1998 \ldots \ldots$. & $10,845-11,138$ & 24 & 276 \\
$1999 \ldots \ldots$. & $11,251-11,299$ & 2 & 22 \\
\hline
\end{tabular}

nights on which observations were made and the number of observations are listed in columns (4) and (5). Note that these numbers do not always total to the corresponding values in Table 2 since some points did not meet the criteria for inclusion in any subset. When possible, we have determined an epoch of maximum light and its estimated uncertainty (col. [6] of Table 3), the amplitude (col. [7]), and the intensity mean magnitude (col. [8]) for each subset. When either maximum or minimum light was not present in our light curve, we have reported the difference between the brightest and faintest observation as a lower limit for the amplitude.

An examination of the data showed that a single period could not be found which was valid over the entire interval of our observations. We have plotted the $O-C$ diagram in Figure 1 using maxima listed in Table 3 . The predicted dates of maximum were determined with a period of 0.44194 days and an epoch of MHJD = 10,705.64 which were derived from the data from 1997 and 1998

TABLE 3

SumMary OF THE SUBSETS OF THE PHOTOMETRY

\begin{tabular}{|c|c|c|c|c|c|c|c|}
\hline $\begin{array}{l}\text { Year } \\
(1)\end{array}$ & $\begin{array}{l}\text { MHJD Interval } \\
\text { (2) }\end{array}$ & $\begin{array}{l}\text { Symbol }^{\mathrm{a}} \\
\text { (3) }\end{array}$ & $\begin{array}{l}\text { Number of Nights } \\
\text { (4) }\end{array}$ & $\begin{array}{c}\text { Number of Observations } \\
\text { (5) }\end{array}$ & $\begin{array}{l}\text { MHJD of Maximum } \\
\text { (6) }\end{array}$ & $\begin{array}{l}\Delta V \\
(7)\end{array}$ & $\begin{array}{c}\langle V\rangle \\
(8)\end{array}$ \\
\hline \multirow[t]{2}{*}{$1991 \ldots \ldots$} & $8102.8-8112.8$ & $\mathrm{a}$ & 4 & 7 & $8110.64 \pm 0.01$ & $>0.15$ & 12.78 \\
\hline & $8149.6-8192.6$ & $\mathrm{~b}$ & 7 & 19 & & 0.27 & 12.82 \\
\hline \multirow[t]{3}{*}{$1995 \ldots \ldots$} & 9871.7-9885.9 & $\mathrm{a}$ & 3 & 53 & $9885.68 \pm 0.01$ & 0.44 & 12.82: \\
\hline & 9958.6-9986.7 & $\mathrm{b}$ & 6 & 277 & $9974.73 \pm 0.03$ & $>0.19$ & 12.84: \\
\hline & $10,002.6-10,002.7$ & c & 1 & 32 & & $>0.19$ & \\
\hline \multirow[t]{5}{*}{$1996 \ldots \ldots$} & $10,233.7-10,238.9$ & a & 2 & 14 & $10,238.73 \pm 0.03$ & $>1.00$ & 12.86: \\
\hline & $10,260.6-10,267.9$ & $\mathrm{~b}$ & 4 & 99 & $10,265.72 \pm 0.01$ & 1.24 & 12.83 \\
\hline & $10,279.6-10,304.7$ & c & 6 & 42 & $10,288.72 \pm 0.01$ & $>1.33$ & 12.82 \\
\hline & $10,320.6-10,332.8$ & d & 2 & 28 & & $>0.82$ & . \\
\hline & $10,340.6-10,357.7$ & $\mathrm{e}$ & 6 & 111 & $10,353.71 \pm 0.01$ & 1.39 & 12.82 \\
\hline \multirow[t]{4}{*}{$1997 \ldots \ldots$} & $10,655.6-10,656.9$ & a & 2 & 32 & $10,655.71 \pm 0.01$ & 0.87 & 12.81 \\
\hline & $10,702.6-10,709.7$ & $\mathrm{~b}$ & 3 & 43 & $10,705.64 \pm 0.01$ & $>0.53$ & \\
\hline & $10,717.6-10,723.7$ & c & 3 & 39 & $10,723.74 \pm 0.03$ & 0.54 & 12.83 \\
\hline & $10,726.5-10,740.7$ & d & 4 & 42 & $10,731.71 \pm 0.01$ & 0.49 & 12.88 \\
\hline \multirow{5}{*}{$1998 \ldots \ldots$} & $10,893.8-10,921.9$ & $\mathrm{a}$ & 8 & 50 & $10,894.81 \pm 0.01$ & 0.73 & 12.82 \\
\hline & $10,950.6-10,985.9$ & $\mathrm{~b}$ & 4 & 96 & $10,950.91 \pm 0.01$ & 0.98 & 12.80 \\
\hline & $11,013.6-11,019.9$ & c & 2 & 18 & $11,013.67 \pm 0.01$ & 1.04 & 12.75 \\
\hline & $11,034.6-11,047.8$ & d & 4 & 72 & $11,047.70 \pm 0.01$ & 0.99 & 12.80 \\
\hline & $11,068.7-11,096.7$ & $\mathrm{e}$ & 4 & 38 & $11,075.53 \pm 0.04$ & $>0.81$ & 12.80: \\
\hline $1999 \ldots \ldots$ & $11,251.8-11,299.9$ & $\mathrm{a}$ & 2 & 22 & $11,299.86 \pm 0.02$ & 0.80 & 12.78 \\
\hline
\end{tabular}

${ }^{\text {a }}$ Symbols in Fig. 2: a, circles; b, triangles with vertex up; c, triangles with vertex down; d, diamonds; e, crosses. 


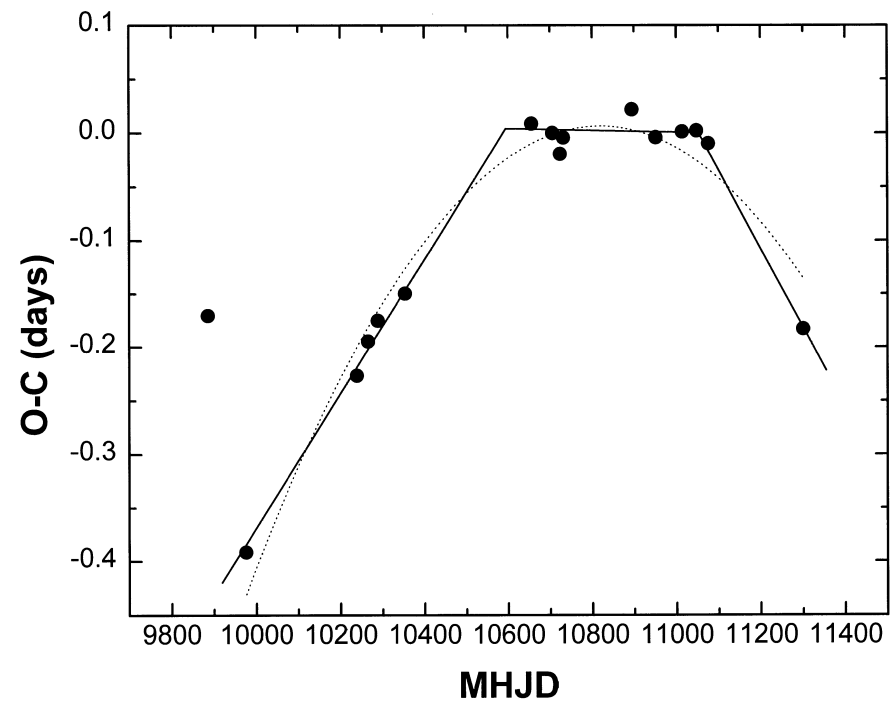

FIG. 1. $-O-C$ plot for maxima listed in Table 3. The first maximum has been omitted. The computed dates of maximum were determined with a period of 0.44194 and an epoch of MHJD $=10,705.64$. The solid lines result from fits based on the assumption of abrupt period changes, while the dotted curve is a fit assuming a steady period increase.

$($ MHJD $=10,655-11,097)$. The maximum from 1991 was omitted from the plot because the gap of 4 years until the next maximum precluded a meaningful cycle count.

Some RR Lyrae stars have been observed to exhibit steadily increasing or decreasing periods over a number of years, while in others the period is constant most of the time with sudden changes. In Figure 1 we show fits to the observed phase shifts using both assumptions (and omitting one point as discussed below). The parabola (dashed line) is a fit for a steady decrease in period, while the series of three straight lines (solid lines) represents the case with sudden period increases. The solid lines clearly represent the better fit to the data. Thus, we will adopt the assumption that the period of V442 Her is stable over intervals of 1 or 2 years with sudden period changes.

The point farthest to the left in Figure 1 appears to be anomalous. To accommodate it we would have to adopt a period of 0.407 days or less prior to MHJD $=9958$. On the other hand, the data from MHJD = 9958-10,003 (the second and third subsets of data from 1995) require a period

TABLE 4

ADOPTED EPHEMERIDES FOR V422 HeR

\begin{tabular}{ccc}
\hline \hline Year & Period & MHJD of Maximum \\
\hline $1991 \ldots \ldots \ldots \ldots$ & $0.4414 \pm 0.00005$ & 8110.64 \\
$1995 / 1996 \ldots \ldots$ & $0.44223 \pm 0.00003$ & $10,288.72$ \\
$1997 / 1998 \ldots \ldots$ & $0.44194 \pm 0.00005$ & $10,705.64$ \\
$1999 \ldots \ldots \ldots \ldots$ & $0.4416^{\mathrm{a}}$ & $11,299.86$ \\
\hline
\end{tabular}

a Ambiguous due to lack of multiple maxima in 1999. See text for discussion. of about 0.442 days, consistent with observations from $1996, \mathrm{MHJD}=10,233-10,358$. A period change of 0.035 days or more during a single observing season is implausibly large. For this reason and because of the light-curve peculiarities discussed below, we have neglected the leftmost point in determining the period of V442 Her. The adopted period which is implied by the fitted line before MHJD 10,560 in Figure 1 is $0.44223 \pm 0.00003$ days.

Unfortunately, failure of the CCD photometry system terminated the observations during the 1999 season, and we were able to obtain only a single epoch of maximum light. Figure 1 shows that this epoch is inconsistent with the 1997/ 1998 period. However, there are unresolved ambiguities. The point and line plotted in Figure 1 for 1999 correspond to a period of 0.4416 days (i.e., a decrease of 0.0003 days) on the assumption that a sudden change occurred at the end of the 1998 season. A larger change and hence shorter period would be obtained if the change had occurred later. On the other hand, if we decreased the cycle count between 1998 and 1999 by one, the derived period would increase to 0.4424 (an increase of 0.0005 ) which is equally plausible. Without further data, we cannot resolve these ambiguities and adopt the shorter period. This has no effect on the discussion below.

Period changes apparently occurred near MHJD 10,560 (between the 1996 and 1997 observing seasons) and after 11,060 (at or after the end of the 1998 observing season). The adopted periods and epochs are listed in Table 4 for the various observing seasons. All these periods were based on the above discussion except that for 1991. That period was determined from a power spectrum analysis of all the data for that year.

The GCVS lists two different periods for V442 Her: 0.4421143 prior to 1957 and 0.4420840 since then. These values fall within the range of periods we have determined, which implies that the period does not systematically decrease over long intervals of time. The fact that the GCVS periods differ by only 0.00003 days while the period changes we have determined are an order of magnitude larger is surprising. It may be a simple coincidence or may indicated that the period was more stable in the past.

In Figure 2 all our photometry for V442 Her is plotted against phase calculated with the appropriate periods and epochs from Table 4. Data from each season is presented as a separate panel. Points from the various subsets defined in Table 3 are denoted by different symbols as indicated in the third column of the table.

\section{DISCUSSION}

If period changes in RR Lyrae stars were produced by stellar evolution, they would generally be very small, much less than a day per million years (Sweigert \& Renzini 1979 

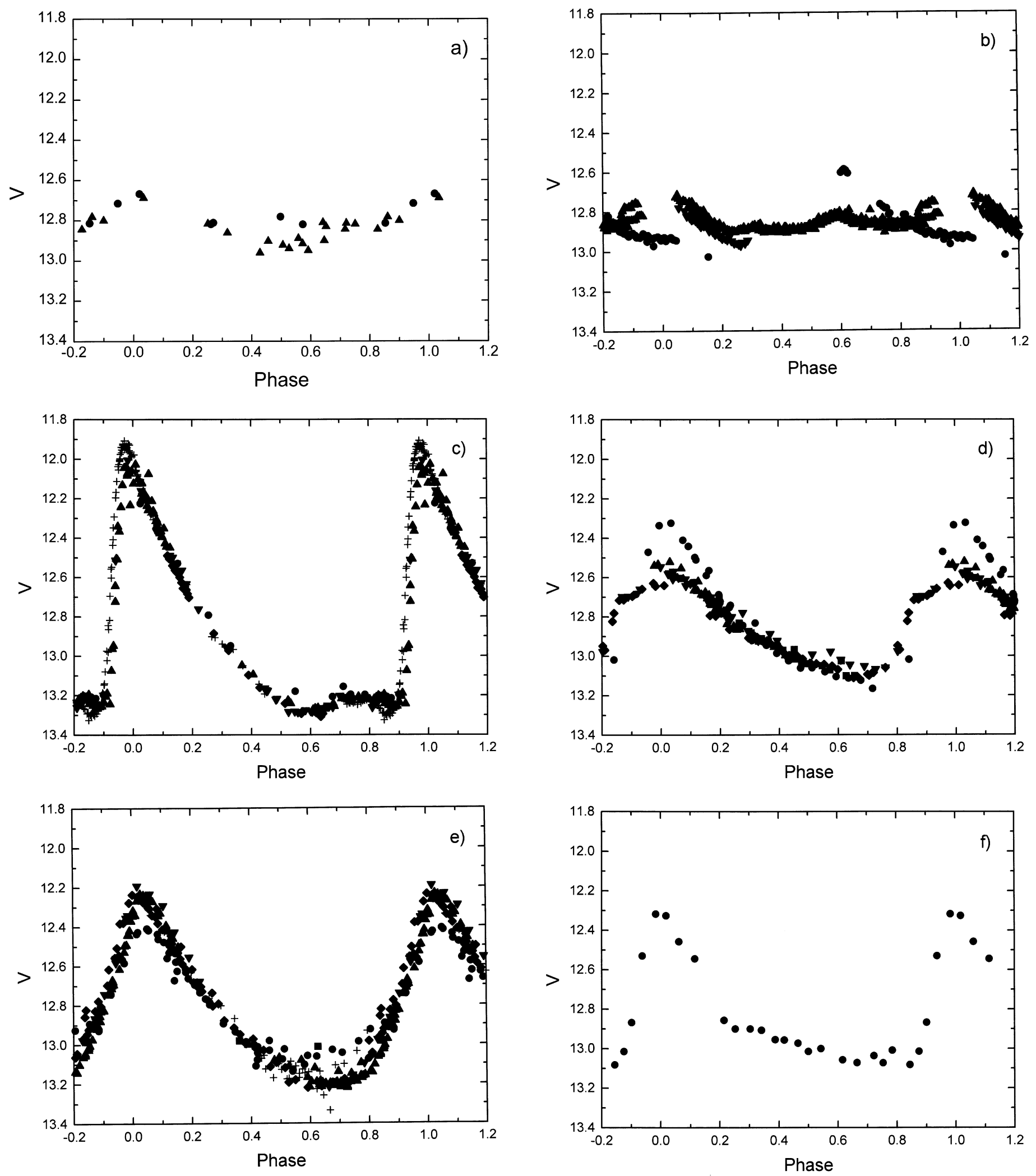

FIG. 2.-Light curves for V442 Her for the years $(a)$ 1990, (b) 1995, (c) 1996, (d) 1997, (e) 1998, and $(f)$ 1999. In each case the symbols denote the data for the subsets from Table 3 as indicated in the table. Squares represent points which are not in any of the subsets of Table 3. The phases used in these plots were calculated from the periods and epochs listed in Table 4. 
and Lee 1991), and might be expected to produce a smooth, continuous increase or decrease in the period. Although many RR Lyrae stars exhibit continuous changes at rates consistent with evolution, there are numerous stars which exhibit larger rates. Some exhibit long intervals of constant period with sudden increases or decreases while others show random changes which are difficult to categorize (see Tsesevich 1972 for a discussion of field stars; Smith 1995 and references therein for stars in clusters; and Sweigert \& Renzini 1979 for a theoretical discussion). In many cases, limited temporal coverage and uncertainties in times of maximum obscure the behavior.

As noted above, during the past five seasons V442 Her has undergone two abrupt period changes of $\Delta P \approx 3 \times 10^{-4}$ days separated by 1.3 years. In a large majority of stars where abrupt period changes have been observed, they are 1 or more orders of magnitude smaller than this [e.g., Tsesevich 1972; Table 4 lists 42 field stars with abrupt period changes in the range $(0.015-0.76) \times 10^{-4}$ days; Storm, Carney, \& Beck 1991 list period changes for 10 stars in M5 which range up to $0.32 \times 10^{-4}$ days; Smith \& Sandage 1981 list period changes for 30 stars in M15 which range up to $\Delta P=0.34 \times 10^{-4}$ days; Clement, Fernace, \& Simon1993 list period changes for 23 stars in M68 which range up to $\Delta P=0.31 \times 10^{-4}$ days; Stagg \& Wehlau 1980 found that $\mathrm{V} 25$ in NGC 6934 had a period increase of $\Delta P \approx 1.6 \times 10^{-4}$ days]. Note that most of these stars exhibit only a single period jump in many decades of monitoring. In those with more than one change, the changes are normally separated by at least 20 years.

Stars which may be similar to V442 Her in regard to their period changes are V104 in $\omega$ Cen (Belserene 1964), SZ Hya (Tsesevich 1972, Fig. 4) and DI Lyr (Tsesevich 1977). $\omega$ Cen V104 may have exhibited period decreases of $\Delta P \approx 4 \times 10^{-4}$ days and $\Delta P \approx 3 \times 10^{-4}$ days separated by about 13 years. However, this is uncertain in as much as the temporal coverage would permit a parabolic fit to the $O-C$ diagram which is nearly as good as several line segments. In the case of SZ Hya, there may have been two period decreases of $\Delta P \approx 1 \times 10^{-4}$ days separated by about 10 years, or, as Tsesevich suggests, the period may have decreased steadily by $2 \times 10^{-4}$ days over 10 years. DI Lyr experienced a period increase of $\Delta P \approx 4 \times 10^{-4}$ days followed by a decrease to the original period 4 years later.

Although we believe that V442 Her exhibits sudden period changes, it is of interest to compare the rate of the steady period change represented by the dashed curve in Figure 1 with the rates for other stars. The rate is often expressed with the parameter $\alpha=(1 / P)(d P / d t)$ which has a value of -448 cycles per million years for V442 Her. Rathbun \& Smith (1997) examined period change data for RR Lyrae stars in seven globular clusters and found that $\alpha$ ranged between -4 and 2.1 cycles per million years. Thus, we conclude again that the period change exhibited by V442 Her is extreme.

As indicated above, any discussion of period changes in RR Lyrae stars is confused by the difficulty of interpreting some of the $O-C$ diagrams. However, it is clear that the size of the period changes in V442 Her and the relatively short interval between them are extreme compared with most other RR Lyrae stars.

As can be seen from Figure 2 and from Table 3, the amplitude of V442 Her undergoes large changes from 1 year to another and significant changes over times of weeks or months. The measured amplitudes and lower limits are plotted against MHJD in Figure 3 (again omitting data from 1991). The two maxima near MHJD 10,350 and 11,000 and the minima at 10,000 and 10,700 suggest a timescale of about 700 days. On the other hand, if the small amplitude in 1991 represents another minimum, it would require a somewhat longer modulation period, about 900 days. We conclude that the amplitude of V442 Her varies irregularly over a timescale somewhat less than a thousand days.

An inspection of Figure 2 shows that the appearance of the light curve changes completely as the amplitude varies. For example, during 1996 (Fig. 2c) when the amplitude was large, the light curve was typical of a Bailey type ab RR Lyrae star. The changing amplitude over the 4 months spanned by the observations is typical of stars exhibiting the Blazhko effect. Similarly, in 1998 (Fig. 2e) the star also exhibited a light curve that would be classed as type ab. However, even though the amplitude was similar in 1996 and 1998 and both times are during an interval of increasing amplitude, there are significant differences in the appearance of the light curve. In 1996 there was a flat minimum lasting about a third of the cycle followed by a rapid rise to maximum in less than $5 \%$ of the cycle. On the

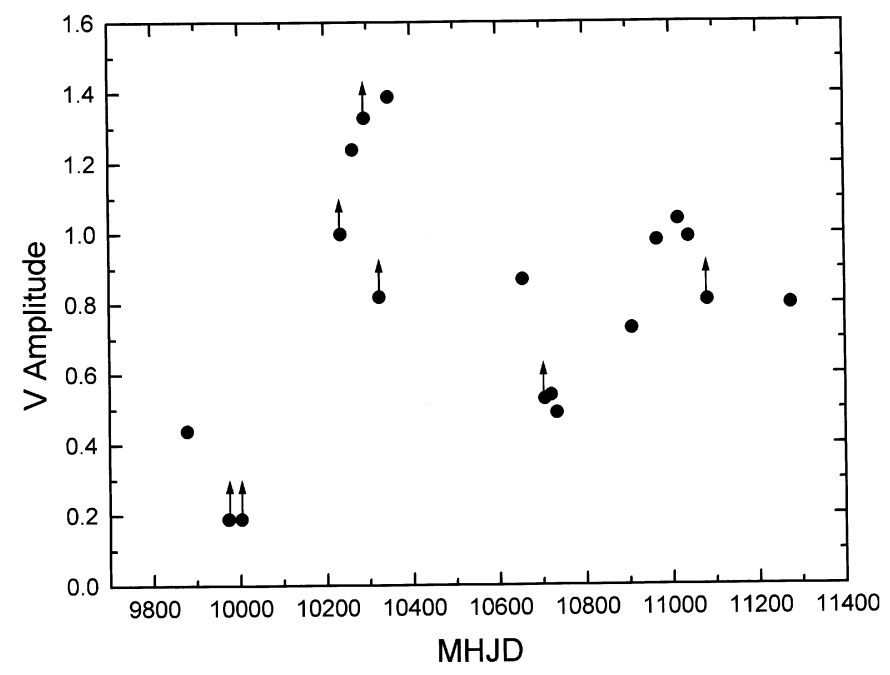

FIG. 3.-The amplitude of V442 Her plotted against MHJD. Data from 1991 are not included in the diagram. Arrows indicate lower limits. 
other hand, in 1998 the minimum was rounded and the rise to maximum occupied about $20 \%$ of the cycle.

During 1991 (Fig. 2a) and 1995 (Fig. 2b), times of small amplitude, the light curves did not resemble an RR Lyrae star at all. Although the variations repeat well from cycle to cycle over a short interval, over a period of weeks there are significant changes. A peculiarity of the 1995 data is the apparent maximum at phase 0.61 and the sequence of points sloping downward to a magnitude of 12.95 at phase 1.0 (Fig. 2b, solid circles). This feature is the source of the anomalous maximum in Figure 1 which was discussed above. These points were taken on three nights in a 2 week interval at the start of the 1995 season with a large majority on a single night (MHJD =9885.67-9885.87). Given the fact that the data from the latter part of 1995 are consistent with the period indicated by the 1996 observations, we speculate that the maximum may, in fact, correspond to the small bump around phase 0.6 in the data from the latter part of the observing season. That would then suggest that a relatively large bump was present at the start of the 1995 observing season and decreased over several months. At the same time, the maximum appears to have increased although it is poorly defined due to the gap near phase 0 .

Theoretical explanations of secondary bumps in the light curves of pulsating stars involve shocks or echos from the core. The presence of a large bump at a time of small overall amplitude is at variance with these models. Thus it would be of great interest to search for this phenomena at future epochs of low amplitude so as to clarify its relationship to the primary maxima. If confirmed, it will provide a challenge to pulsation theory.

Finally, we note that during 1997 (Fig. 2d) the star was at an intermediate, decreasing amplitude. While the earliest data in that season exhibit a type ab light curve similar to that observed in 1998, by the end of the season the top of the light curve appears to be truncated. This produces a humped light curve reminiscent of those shown for type II Cepheids by Diethelm (1983, his type BL). This behavior is unknown among RR Lyrae stars and appears to represent a stage in the transition between typical RR Lyrae behavior and the peculiar small amplitude behavior.

In our 1996 light curve (Fig. 2c) the latest data (MHJD $=10,340-10,358$, crosses) leads the data from 70 to 80 days earlier $(\mathrm{MHJD}=10,260-10,268$, triangles with vertex up) during rising light by about 0.02 cycles. Similar phase shifts are apparent in the 1998 data (Fig. 2e). Phase changes relative to a long-term mean period are relatively common in Blazhko stars (Lee 1997). For example, in RR Lyrae itself, the phase of maximum drifts by about 0.05 cycles over the Blazhko cycle (see the light curves shown by Walraven 1949).

The last column of Table 3 lists the intensity mean magnitude of V442 Her during the various intervals of the observations. The reliability of these numbers is strongly influenced by the phase coverage and some are marked as uncertain for that reason. A close examination of all the data shows that nearly all values of $\langle V\rangle$ given in the table are consistent with the hypothesis that as V442 Her experiences its large light-curve modulations the mean magnitude remains constant. As would be expected, the $\langle V\rangle-\langle R\rangle$ colors are even more constant so we conclude that the modulation does not generally affect the mean luminosity of the star. The only exceptions are the mean magnitudes for MHJD 10,717.6-10,723.7 and 10,726.5-10,740.7. In Figure $2 d$ it can be seen that the maxima in both those intervals, denoted by triangles with vertex down and diamonds respectively, do not reach the level of the previous welldefined maximum (circles) while the brightness around minimum is similar, particularly in the 10,726.5-10,740.7 interval. This indicates that we observed a genuine diminution in the luminosity of the star by about $5 \%$ at the end of the observing season. Thus, the mechanism which produced the apparent chopping off of the peak of the light curve redistributed a significant amount of energy and there should be a subsequent increase in the luminosity to maintain long-term equilibrium. Unfortunately, the next data were 150 days later, and the star had apparently returned to its equilibrium luminosity by then.

\section{CONCLUSIONS}

The Blazhko effect in RR Lyrae stars can produce changes in amplitude, phase drift, and changes in the shape of the light curve (see, for example, a description of the effect by Smith 1995 and an example of light curves in Walraven 1949). All these are present in V442 Her. However, while the phase drift we have found is similar in size to many stars exhibiting the Blazhko effect, the large amplitude modulation, the long period of the modulation and the radical changes in the form of the light curve are extreme compared to other known Blazhko stars. Stars which have been studied in detail show amplitudes which typically vary by up to a half a magnitude over the Blazhko cycle (see, for example, Lee 1997 and Walraven 1949). An extreme example is WY Dra (Chis, Chis, \& Mihoc 1975) where the amplitude ranges over nearly a magnitude. However, it is never less than about 1.2 mag. In contrast, the amplitude of V442 Her has varied from 0.27 to $1.39 \mathrm{mag}$, a range of 1.12 mag, during our observations. Additionally, modulation occurs over a timescale of the order of 700 days or more while the longest known Blazhko cycle is about 530 days in RS Boo (Oosterhoff 1946; Kanyo 1980; Firmanyuk 1988; note that a small modulation of the pulsation may also occur on a 48 day timescale). Finally we note that the lightcurve form for V442 Her ranges from that of a typical Bailey type ab star at large amplitude to one which bears no resemblance to an RR Lyrae light curve during times of low amplitude. Again, this is not true of other RR Lyrae. For 
example, Teays (1993) used Fourier decomposition to show that as RR Lyrae goes through its Blazhko cycle, the lightcurve shape always matches other stars of similar period. We conclude that if the behavior of V442 Her is due to the Blazhko effect, it is perhaps the most extreme example of this behavior known.

It is of interest to ask whether there are other stars which behave similarly to V442 Her. Microlensing surveys are a rich source of information on unusual behavior in pulsating variables. For example, in the Large Magellanic Cloud, Alcock et al. (1996) demonstrated the presence of a significant number of RR Lyrae stars pulsating in the second harmonic mode while Alcock et al. (2000) found that about $14 \%$ of RRc stars are double-mode pulsators. However, no stars similar to V442 Her have come to light in the microlensing data.

The data of Corwin, Carney, \& Nifong (1999) for the RR Lyrae star V20 in NGC 5466 showed intervals with no pulsation during some of their observations. However, this star appeared to change from a state with no variation to normal pulsation from one night to the next. Given this and its short period, 0.23 days, it does not appear that V20 is similar to V442 Her.

Other stars which might be similar to V442 Her are V79 in M3 $(P=0.48$ days $)$ and V15 in NGC $6121(P=0.44$ days). Clement \& Goranskij (1999) found that before 1992 V19 had an irregular light curve but since has become a double-mode pulsator. On the other hand, Clementini et al.
(1994) found that V15 may be changing from an RRab to an RRc star. They suggested that these two stars might belong to a new class of variables which they dubbed "switchingmode pulsators." Although there are some differences in behavior, we suggest that V442 Her could be a third member of this group.

As demonstrated above, V442 Her exhibits unusual behavior which makes it an interesting object for further study. In particular, it may offer insights into the Blazhko effect or into mode switching. Broad band photometry must be continued to clarify the long-term behavior and to show whether there is a stable modulation period present. Spectroscopy or narrowband photometry would be useful to determine where V442 Her falls in the metallicity range of RR Lyrae stars. Finally, as observational tests are devised for various explanations of the Blazhko effect, V442 Her should clearly be included due to its extreme behavior.

\section{ACKNOWLEDGMENTS}

The instrumentation used for the observations described in this paper was funded by the National Science Foundation (grant AST 85-04072) and some of the observations were conducted with support from NSF grant AST 8815806. Support for publication expenses was provided by the American Astronomical Society through its small grants program.

\section{REFERENCES}

Alcock, C., et al. (The MACHO Collaboration). 1996, AJ, 111, 1146

.2000, ApJ, in press

Belserene, E. P. 1964, AJ, 69, 475

Breger, M. 1990, Inf. Bull. Variable Stars 3422

Chis, D., Chis, D., \& Mihoc, I. 1975, Inf. Bull. Variable Stars 960

Clement, C. M., Fernace, S., \& Simon, N. R. 1993, ApJ, 412, 483

Clement, C. M., \& Goranskij, V. P. 1999, ApJ, 513, 767

Clementini, G., Merighi, R., Pasquini, L., Cacciari, C., \& Gouiffes, C. 1994, MNRAS, 267, 83

Corwin, T. M., Carney, B. W., \& Nifong, B. G. 1999, AJ, 118, 2875

Diethelm, R. 1983, A\&A, 124, 108

Firmanyuk, B. N. 1988, Inf. Bull. Variable Stars 3259

Kanyo, S. 1980, Inf. Bull. Variable Stars 1832

Kholopov, P. N. 1985, General Catalogue of Variable Stars (4th ed.; Moscow: Nauka)

Lee, K. M. 1997, Ph.D. thesis, Univ. Nebraska
Lee, Y.-W. 1991, ApJ, 367, 524

Oosterhoff, P. Th. 1946, Bull. Astron. Inst. Netherlands, 10, 101

Rathbun, P. G., \& Smith, H. A. 1997, PASP, 109, 1128

Schmidt, E. G. 1991, AJ, 102, 1766

Schmidt, E. G., \& Reiswig, D. E. 1993, AJ, 106, 2429

Smith, H. A. 1995, RR Lyrae Stars (Cambridge: Cambridge Univ. Press)

Smith, H. A., \& Sandage, A. 1981, AJ, 86, 1870

Stagg, C., \& Wehlau, A. 1980, AJ, 85, 1182

Storm, J., Carney, B. W., \& Beck, J. A. 1991, PASP, 103, 1264

Sweigert, A. V., \& Renzini, A. 1979, A\&A, 71, 66

Teays, T. J. 1993, in New Perspectives on Stellar Pulsation and Pulsating Variable Stars, ed. J. M. Nemec \& J. M. Matthews (Cambridge: Cambridge Univ. Press), 410

Tsesevich, V. 1972, Vistas Astron., 13, 241

- 1977, Inf. Bull. Variable Stars 1370

Walraven, Th. 1949, Bull. Astron. Inst. Netherlands, 11, 17 